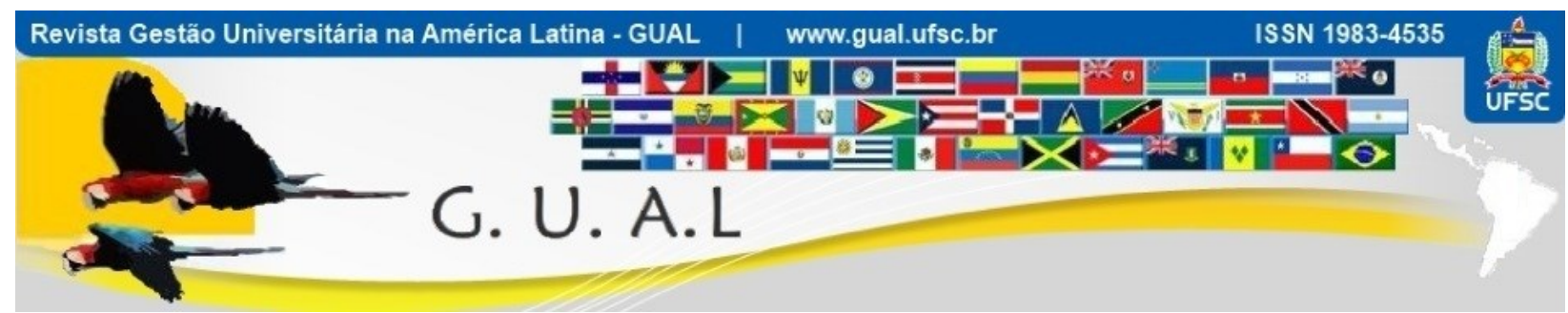

DOI: https://doi.org/10.5007/1983-4535.2021.e74569

\title{
O OLHAR DOCENTE E DISCENTE SOBRE RESPONSABILIDADE SOCIAL UNIVERSITÁRIA
}

\section{THE VIEW OF PROFESSORS AND STUDENTS ON UNIVERSITY SOCIAL RESPONSIBILITY}

\author{
Elise Cristina Eidt, Mestre \\ https://orcid.org/0000-0001-7938-7970 \\ elise.eidt@uffs.edu.br \\ Universidade Federal da Fronteira Sul | Campus Chapecó \\ Chapecó | Santa Catarina | Brasil \\ Rosane Calgaro, Doutora \\ https://orcid.org/0000-0002-8487-2652 \\ rocalgaro@yahoo.com.br \\ Universidade Estadual do Oeste do Paraná | Campus Francisco Beltrão \\ Francisco Beltrão | Paraná | Brasil \\ Larissa de Lima Trindade, Doutora \\ https://orcid.org/0000-0002-9708-0363 \\ larissa.trindade@uffs.edu.br \\ Universidade Federal da Fronteira Sul | Campus Chapecó \\ Chapecó | Santa Catarina | Brasil \\ Jucelia Appio Frizon, Doutora \\ https://orcid.org/0000-0002-2721-4418 \\ juceliaappio@yahoo.com.br \\ Universidade Estadual do Oeste do Paraná | Campus Francisco Beltrão \\ Francisco Beltrão | Paraná | Brasil
}

Recebido em 07/junho/2020

Aprovado em 26/novembro/2020

Publicado em 01/janeiro/2021

Sistema de Avaliação: Double Blind Review

Agradecimentos: Programa de Bolsas Universitárias de Santa Catarina (UNIEDU)

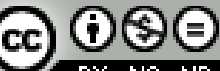

Esta obra está sob uma Licença Creative Commons Atribuição-Uso. 


\title{
RESUMO
}

Este artigo apresenta um estudo sobre a Responsabilidade Social Universitária (RSU) na visão de docentes e discentes de uma Universidade Federal. Tendo em vista a natureza da universidade, o estudo foi desenvolvido com base no modelo de análise da RSU proposto por Vallaeys, Cruz e Sasia (2009). Foram abordados os eixos Campus Responsável, Formação Profissional e Cidadã, Gestão Social do Conhecimento e Participação Social. A base teórica discute brevemente o modelo utilizado para análise. O delineamento metodológico adotado caracteriza um estudo descritivo quantitativo, tendo como unidade de análise a Universidade Federal da Fronteira Sul (UFFS). Para isso um questionário foi aplicado a fim de verificar se o modelo usado descreve a concepção dos docentes e discentes a respeito da RSU praticada na UFFS. Os resultados da regressão múltipla revelam que, apesar de terem percepções diferentes sobre o compromisso social desenvolvido pela Instituição, docentes e discentes compreendem o compromisso social a partir do Eixo Campus Responsável, que implica em integrar as preocupações de responsabilidade social de forma transversal na estratégia e na matriz base das atividades desenvolvidas.

Palavras-chave: Campus Responsável. Modelo de Análise da RSU. Compromisso Social. Universidade Pública Federal.

\begin{abstract}
This article presents a study on the University Social Responsibility (USR) in the view of faculty members and students of a Federal University. Bearing in mind the university's nature, the study was developed based on the model of the USR proposed by Vallaeys, Cruz, and Sasia (2009). Responsible Campus, Professional Formation and Citizen, Social Management of Knowledge, and Social Participation axes were approached. The theoretical basis briefly discusses the model used for analysis. The methodological rendition characterizes a quantitative descriptive study, comprehending the Fronteira Sul Federal University (UFFS) as a unity of analysis. To this end, a questionnaire was applied to check if the model that was used described the conception of faculty and students concerning USR practiced at UFFS. The results of the multiple regression reveal that despite having different perceptions about the social commitment developed by the Institution, both faculty and students perceive the social commitment as of the Axis Responsible Campus, which implies integrating the social responsibilities concerns in a transversal mean and on the base matrix of the developed activities.
\end{abstract}

Keywords: Responsible Campus. Analysis Model of USR. Social Commitment. Federal Public University. 


\section{INTRODUÇÃO}

Desde o seu surgimento, no século XII, as universidades são caracterizadas por desempenhar determinado papel na sociedade. No início, elas atendiam a poucos, pautadas no caráter elitista (BOHRER et al., 2008), entretanto, com o decorrer dos anos, o papel dessas instituições foi se convertendo em uma ideia que ia além da guarda e transmissão do saber, visando a formação da elite pensante, já que, segundo Oliven (2002) e Danesi e Siqueira (2014), o foco inicial era o ensino, com a transferência de saberes, sem ter compromisso com a produtividade material e a abertura da universidade.

Sob a perspectiva histórica, Reis (2007) afirma que as universidades vêm expondo a contemporânea mobilização em torno do seu papel social, considerando a premissa de que, por portarem autonomia na gestão político-pedagógica e de aplicação de recursos, devem estar focadas na resolução de problemas da sociedade. Com essa perspectiva, a atuação das universidades, no cenário da Responsabilidade Social (RS), vem se intensificando, estando relacionada diretamente à discussão sobre a redefinição de papéis e abrangência de suas atividades, que há muito tempo era demandada pela sociedade.

Foi com o advento da extensão que a universidade passou a ampliar sua atuação, estabelecendo, além do ensino e da pesquisa, uma relação mais direta com outros segmentos da sociedade (PUCCI, 1991), possibilitando com isso a estruturação da ideia de compromisso social, o que significa falar de seus deveres e obrigações, dos encargos inerentes à natureza institucional (CALDERÓN, 2005; CRUZ; PASSOS, 2010; CARDENUTO et al., 2017).

Em decorrência da intensificação do compromisso social, a RS ganha novos contornos e relevância, no cenário brasileiro, com a operacionalização do Sistema Nacional de Avaliação da Educação Superior (SINAES), instituído pela Lei no 10.861 de 2004, que está pautado no "[...] aprofundamento dos compromissos e responsabilidades sociais das IES" (BRASIL, 2004, Art. $1^{\circ}, \S 1^{\circ}$ ), constando entre os princípios fundamentais a responsabilidade social com o avanço da qualidade da educação superior.

Os estudos demonstram que as IES precisam assumir como função o desenvolvimento e a disseminação da Responsabilidade Social Universitária (RSU), aliado aos desafios práticos que se visualiza na gestão (ROSETTO, 2011; GOMEZ; PRECIADO, 2013; LO et al.; 2017). Nesta direção, este estudo visa descrever a concepção dos docentes e discentes da Universidade Federal da Fronteira Sul (UFFS) a respeito da Responsabilidade 
Social praticada pela Instituição utilizando como base o Modelo de RSU proposto por Vallaeys, Cruz e Sasia (2009).

Tal modelo retrata a RSU como um eixo transversal e integral em todos os âmbitos da universidade, envolvendo o campus responsável (gestão), a formação profissional e cidadã (ensino), a gestão social do conhecimento (pesquisa) e a participação na sociedade (extensão) (VALLAEYS; CRUZ; SASIA, 2009; VALLAEYS, 2016). A efetivação da responsabilidade social está atrelada ao diálogo participativo com os públicos interno e externo, o que convida permanentemente à autorreflexão da comunidade acadêmica sobre os seus pressupostos epistêmicos e as implicações da sua ação (NASCIMENTO et al., 2015; VALLAEYS, 2017).

A UFFS foi criada em 15 de setembro de 2009, pela Lei no 12.029 , e implantada na Mesorregião Grande Fronteira do Mercosul (MESOMERCOSUL), que abrange mais de 400 municípios. Com sede no Município de Chapecó, Estado de Santa Catarina (SC), e com atuação multicampi em Cerro Largo, Erechim e Passo Fundo, no estado do Rio Grande do Sul (RS); Laranjeiras do Sul e Realeza, no Paraná (PR). A instituição simboliza uma conquista para os movimentos sociais, as lideranças políticas, empresariais e comunitárias engajadas na sua criação, que reivindicavam uma universidade pública na perspectiva de inclusão escolar e superação das desigualdades regionais, e a comunidade acadêmica responsável pela sua implantação (UFFS, 2020).

Nossos estudos revelam dificuldades, quanto à implantação, à construção e à estruturação da RS em instituições de ensino superior. A temática é considerada nova, pois não possui indicadores padronizados, além de ser muito vinculada à mera reprodução da Responsabilidade Social Empresarial. Assim, esta pesquisa contribui para o entendimento e reconhecimento da Responsabilidade Social Universitária, vinculada a todos os âmbitos institucionais (gestão, ensino, pesquisa e extensão), auxiliando na ampliação do uso do Modelo de RSU no Brasil, especialmente por meio de métodos quantitativos de análise.

\section{O MODELO DE RSU DE VALLAEYS, CRUZ E SASIA (2009)}

Acompanhando a evolução dos conceitos de Responsabilidade Social e Responsabilidade Social Universitária, surgiram vários modelos de relatórios e de indicadores. Entre eles, destaca-se um dos modelos pioneiros latino-americano: o modelo de RSU proposto por Vallaeys, Cruz e Sasia em 2009, a partir do Manual de primeros passos.

Salienta-se que este Manual foi financiado pelo Banco Interamericano de Desenvolvimento (BID), e traz uma proposta de ferramenta para o monitoramento de 
indicadores de desempenho em responsabilidade social universitária, os quais integram o autodiagnostico institucional (SANTELI; MONTOYA, 2017). Os indicadores propostos são agrupados de acordo com o âmbito dos impactos que produzem.

Conforme abordam Vallaeys, Cruz e Sasia (2009) e Vallaeys (2016), a RSU abrange para o que as universidades são responsáveis, por quem são responsáveis e como elas são responsáveis. Inicialmente, as IES são responsáveis por colocar a ética no cerne da vida acadêmica e institucional. Como segundo ato, são responsáveis com os diferentes integrantes da comunidade universitária no que refere a aspectos laborais, ambientais e comunidade regional. Em terceiro lugar, são responsáveis pelo desenvolvimento das atividades em áreas como gestão, ensino, pesquisa e comunidade, orientando a produção do conhecimento e os processos de ensino-aprendizagem. Por fim, as universidades devem ter ainda a habilidade de gerir valores humanos, democráticos, sociais e ecológicos.

Com isso, os autores compreendem que a universidade gera quatro tipos de impactos, que podem ser descritos como: i) Impactos do funcionamento organizacional: como qualquer organização trabalhista, a universidade gera impactos nos aspectos laborais, ambientais, nas vidas de seus funcionários administrativos, docentes e estudantes e também poluição em seu ambiente; ii) Impactos educacionais: a universidade tem um impacto direto na formação de jovens e profissionais, bem como na sua maneira de entender e interpretar o mundo, ou seja, tudo o que está relacionado aos processos de ensino-aprendizagem e na construção curricular que derivam no perfil do egresso; iii) Impactos cognitivos e epistemológicos: a universidade coordena a produção de conhecimentos e tecnologias e influencia a definição do que é chamado socialmente de "verdade, ciência, racionalidade, legitimidade, utilidade, educação etc." e iv) Impactos sociais: a universidade tem impacto na sociedade e no seu desenvolvimento econômico, social e político. Não só tem um impacto direto no futuro, pois molda profissionais e líderes, mas é também uma referência e um ator social que pode promover (ou não) o progresso, e ter (ou não) capacidade de ser um interlocutor válido na solução de problemas.

Como expõem Vallaeys (2017), ao determinar quais são os tipos de impactos gerados pela universidade nas suas atividades cotidianas, também será possível perceber os riscos que estão associados a esses impactos, a partir disso a universidade pode refletir e promover iniciativas a favor de impactos positivos, considerando o envolvimento de todos os atores universitários (DUEÑAS, 2015), por meio do desenvolvimento de quatro eixos de 
responsabilidade social da universidade (VALLAEYS, CRUZ; SASIA, 2009; ORSIES, 2018):

1. Campus Responsável: implica a gestão socialmente responsável da organização e seus procedimentos institucionais; do clima de trabalho; da gestão de recursos humanos e desenvolvimento de políticas inclusivas; dos processos democráticos e governança transparente; da justiça e equidade nas políticas de acesso; do cuidado com o meio ambiente, com a criação e manutenção de um campus ambientalmente sustentável, seguro e saudável e ainda do uso de uma comunicação e marketing socialmente responsável;

2. Formação profissional e cidadã: é a gestão socialmente responsável da formação acadêmica e sua relação com os problemas econômicos, sociais, políticos e ecológicos da sociedade e o contato com os atores externos; envolve ainda a formação com pensamento crítico e autônomo, a promoção da empregabilidade, a integração em projetos sociais e voluntariado solidário e a promoção da mobilidade e da colaboração, nacionais e internacionais;

3. Gestão social do conhecimento: é a gestão socialmente responsável da produção e disseminação de conhecimento, pesquisa e modelos epistemológicos promovidos em sala de aula e com parceiros externos, a fim de articular a produção de conhecimento com a agenda de desenvolvimento local e nacional e com os programas sociais;

4. Participação social: é a gestão socialmente responsável da participação da universidade na comunidade. O objetivo é realizar projetos com outros atores de forma que sejam estabelecidos vínculos (criação de capital social na comunidade) para a aprendizagem mútua e o desenvolvimento social.

A partir desse contexto, Vallaeys, Cruz e Sasia (2009) propõem um Manual de primeros pasos que serve como um processo de diálogo e autodiagnóstico para que as instituições integrem suas diferentes áreas e funções e colaborem de forma coerente com o desenvolvimento do ambiente onde estão inseridas. Inclui ainda instrumentos para identificar áreas de intervenção e medidas para melhorar suas atividades diárias de gestão, ensino, pesquisa e extensão.

Em revisão sistemática e análise bibliométrica da produção científica relacionada com a RSU, realizada na base de dados Web of Science e Scopus, no período compreendido entre 2001 e 20 de junho de 2019, por meio da pesquisa do termo "University Social Responsibility", Duque e Cervantes-Cervantes (2019) identificaram que Vallaeys é o autor mais citado e que o documento com maior impacto na questão da RSU é "Responsabilidad Social Universitaria: manual de primeros pasos", publicado por Vallaeys, Cruz e Sasia em 2009. 
Dentre os estudos realizados que indicaram e/ou aplicaram o modelo teórico de Vallaeys, Cruz e Sasia, destaca-se as revisões bibliográficas sobre RSU de Quezada (2011), Dueñas (2015) e Villalonga e Paspuel (2015). Já Kiszner (2018), em sua dissertação, estudou a RSU em instituições comunitárias de educação superior e, dentre as propostas, elencou uma adaptação do Sistema de Gestão RSU e sugeriu os formulários e instrumentos para o diagnóstico de RSU adaptados para o contexto do Brasil.

Cespédes Aguirre (2019) utilizou o modelo aplicando o grupo de foco para representantes internos e externos, visando conhecer a percepção deles sobre a RSU da Universidad Nacional Hermilio Valdizán de Huánuco. Gómez, Alvarado e Pujol (2018) realizaram um estudo sobre o tema, através da aplicação de questionário ajustado ao modelo, especificamente desde a perspectiva dos públicos internos de uma universidade privada em Porto Rico. Mebarack e Ruiz (2018) trataram do caso da Universidad Tecnológica de El Salvador (UTEC), que desde 2015 adotou um modelo de RSU baseado no Manual de primeros pasos, para desenvolver ações internas e externas. Santeli e Montoya (2017) analisaram os pressupostos teóricos e práticas de RSU em universidades do Distrito Metropolitano de Quito (DMQ), empregando o Manual para realização de revisão bibliográfica, documental, entrevistas em profundidade e revisão das páginas web das instituições. Como observado, o Manual possui reconhecimento, existindo amplo campo de pesquisa nacional sobre a temática da RSU ao olhar de Vallaeys, Cruz e Sasia (2009).

\section{METODOLOGIA}

A fim de conhecer o olhar dos docentes e discentes da UFFS a respeito da responsabilidade social universitária, tendo por base o modelo de Vallaeys, Cruz e Sasia (2009), optou-se por uma análise de abordagem quantitativa (RICHARDSON, 1999). O delineamento adotado caracteriza-se como descritivo (GIL, 2010).

Concernente aos procedimentos, foram empregadas como técnicas de coleta de dados a análise documental e a aplicação de questionários. Como documentos foram analisados os Projetos Pedagógicos Curriculares (PPCs) e o relatório da II COEPE - Conferência de Ensino, Pesquisa e Extensão da UFFS. O questionário teve por base o modelo de diagnóstico proposto por Vallaeys, Cruz e Sasia (2009), composto por quatro eixos de RSU: campus responsável; formação profissional e cidadã; gestão social do conhecimento e participação social. 
Para definir a amostra discente e docente foi aplicada a técnica de amostragem aleatória simples sem reposição (MARCONI; LAKATOS, 2002). O sorteio dos indivíduos que participaram da aplicação do questionário foi feito por meio das listas de alunos e docentes obtidas na Pró-Reitoria de Graduação. Para a amostra intencional considerou-se os cursos de graduação e pós-graduação stricto sensu mais representativos da UFFS. Assim, foram selecionados dois alunos por ano/semestre de ingresso dos dois cursos de graduação (um bacharelado e uma licenciatura) mais representativos dos campi Cerro Largo, Erechim, Laranjeiras do Sul e Realeza. No Campus Chapecó foram escolhidos dois alunos por ano/semestre de ingresso dos quatro cursos de graduação (dois de bacharelado e dois de licenciatura) mais representativos. A justificativa para escolher o dobro de cursos em Chapecó, se dá pelo fato de ser o maior campus e também por possuir maior quantitativo de cursos de graduação (13 no total). Por fim, para o Campus Passo Fundo, como apenas existe a oferta do curso de Medicina, foram selecionados dois alunos por ano/semestre de ingresso desse curso.

Para além dos alunos da graduação, foram selecionados também dois alunos de mestrado do curso mais representativo (em termos de alunos matriculados) em cada um dos campi da UFFS (quando havia oferta de mestrado). Em Chapecó, da mesma forma como se estabeleceu com os graduandos, foi selecionado o dobro de cursos stricto sensu (dois, no caso), por ser o campus com maior oferta de mestrados.

No que se refere aos docentes, foram selecionados inicialmente $50 \%$ dos docentes dos cursos detalhados. Tal decisão permitiu que, se a metade dos sorteados não respondessem ao questionário, houvesse a mesma quantidade de professores para uma segunda rodada.

A Tabela 1 traz a relação dos cursos de graduação e pós-graduação mais representativos em 2018, por campus, e as amostras discentes e docentes estabelecidas.

Os questionários estruturados, conforme eixos e temas detalhados no Quadro 1, foram encaminhados via correio eletrônico ao público-alvo, com a disponibilização de link do Google Forms para acesso e preenchimento no período de 20 de outubro a 9 de novembro de 2018 para os docentes e de 20 de outubro a 30 de novembro de 2018 para os discentes. Ressalta-se que o questionário foi enviado para os sorteados aleatórios a cada cinco dias, disponibilizando quatro dias para preenchimento. A opção por essa ferramenta para o desenvolvimento e a aplicação dos questionários proporcionou agilidade e rapidez na execução da coleta de dados da pesquisa. Para estruturação dos questionários utilizou-se da 
escala Likert de 5 pontos: 1. Discordo totalmente; 2. Discordo parcialmente; 3. Nem concordo nem discordo; 4. Concordo parcialmente e 5. Concordo totalmente.

Tabela 1 Cursos de graduação e pós-graduação da UFFS, por campus, e amostras de discentes e docentes

\begin{tabular}{|c|c|c|c|}
\hline Campus & Curso & $\begin{array}{c}\text { Amostra } \\
\text { discentes* }\end{array}$ & $\begin{array}{c}\text { Amostra } \\
\text { docentes }(50 \%)\end{array}$ \\
\hline \multirow{6}{*}{ Chapecó } & Agronomia & 22 & 13 \\
\hline & Enfermagem & 20 & 20 \\
\hline & História & 28 & 08 \\
\hline & Letras & 26 & 15 \\
\hline & Programa de Pós-Graduação em Educação & 02 & $* * *$ \\
\hline & Programa de Pós-Graduação em História & 02 & $* * *$ \\
\hline \multirow{3}{*}{ Cerro Largo } & Agronomia & 24 & 11 \\
\hline & Letras (Português e Espanhol) & 20 & 10 \\
\hline & Prog. de Pós-Grad. em Ambiente e Tec. Sustentáveis & 02 & $* * *$ \\
\hline \multirow{3}{*}{ Erechim } & Agronomia & 24 & 15 \\
\hline & Pedagogia & 18 & $10 * *$ \\
\hline & Prog. de Pós-Grad. Profissional em Educação & 02 & $* * *$ \\
\hline \multirow{3}{*}{$\begin{array}{l}\text { Laranjeiras do } \\
\text { Sul }\end{array}$} & Agronomia & 24 & 15 \\
\hline & Educação do Campo & 28 & $18 * *$ \\
\hline & Prog. de Pós-Grad. em Agr. e Desenv. Rural Sust. & 02 & $* * *$ \\
\hline Passo Fundo & Medicina & 20 & 31 \\
\hline \multirow{5}{*}{ Realeza } & Licenciatura em Ciências Biológicas & 22 & $11 * *$ \\
\hline & Medicina Veterinária & 30 & $11 * *$ \\
\hline & Prog. de Pós-Grad. em Saúde, Bem-Estar Animal e & & \\
\hline & Produção Animal Sustentável na Fronteira Sul & 02 & $* * *$ \\
\hline & Total Amostra & 318 & 188 \\
\hline
\end{tabular}

Fonte: Elaborada pelas autoras a partir dos dados abertos da UFFS (2018a) e UFFS (2018b).

*Para a definição da amostra foram considerados o ano/semestre em que havia pelo menos dois alunos matriculados. O semestre em que havia apenas um aluno matriculado, foi remanejado para o semestre seguinte (acima).

** Amostra arredondada para cima.

***Docentes atuam na graduação e pós-graduação

Após a coleta, foram obtidas um total de 133 respostas válidas ao questionário dos discentes e 65 respostas ao questionário docente. Para avaliar os dados e sintetizá-los para cada um dos eixos de RSU, foi utilizado como método a análise a regressão múltipla, sendo a análise realizada com o IBM Statistical Package for the Social Sciences (SPSS) 19.

Primeiramente, procedeu-se análise descritiva com apresentação das variáveis com maiores e menores médias na visão dos docentes e dos discentes. Em seguida, para a construção das regressões múltiplas - uma com a visão dos docentes e outra com a visão dos discentes sobre a responsabilidade social universitária praticada pela UFFS a partir do Modelo de Vallaeys, Cruz e Sasia (2009) - foram realizados testes para verificar se as medidas dos construtos, propostos a partir do Modelo, eram significativamente correlacionadas, para isso foram realizados o Teste de Kaiser-Meyer-Olkin (KMO) e esfericidade de Bartlett. 
Quadro 1 Eixos e temas a observar no estudo

\begin{tabular}{|c|c|c|c|}
\hline Eixos & Temas a observar & Discentes & Docentes \\
\hline $\begin{array}{c}\text { Campus } \\
\text { Responsável }\end{array}$ & $\begin{array}{l}\text { 1. Direitos humanos, equidade de gênero e não discriminação. } \\
\text { 2. Desenvolvimento pessoal e profissional, bom clima laboral e aplicação } \\
\text { dos direitos trabalhistas. } \\
\text { 3. Meio ambiente (campus responsável). } \\
\text { 4. Transparência e democracia (boa gestão). } \\
\text { 5. Comunicação e divulgação responsável. }\end{array}$ & $\begin{array}{l}\text { Questões } \\
1 \text { a } 20\end{array}$ & $\begin{array}{l}\text { Questões } \\
1 \text { a } 20\end{array}$ \\
\hline $\begin{array}{c}\text { Formação } \\
\text { profissional e } \\
\text { cidadã }\end{array}$ & $\begin{array}{l}\text { 1. Inclusão social. } \\
\text { 2. Presença de temáticas cidadãs e de responsabilidade social nos } \\
\text { projetos curriculares (desenvolvimento sustentável, ética profissional e } \\
\text { cívica, gestão da RS, entre outros). } \\
\text { 3. Integração de atores sociais externos no desenho das matrizes } \\
\text { curriculares. } \\
\text { 4. Articulação entre profissionalização e voluntariado solidário e a } \\
\text { relevância social da educação. }\end{array}$ & $\begin{array}{l}\text { Questões } \\
21 \text { a } 30\end{array}$ & $\begin{array}{l}\text { Questões } \\
21 \text { a } 30\end{array}$ \\
\hline $\begin{array}{l}\text { Gestão social do } \\
\text { conhecimento }\end{array}$ & $\begin{array}{l}\text { 1. Integração dos atores sociais externos nas pesquisas e nos desenhos } \\
\text { das linhas de investigação. } \\
\text { 2. Difusão, transferência e troca de conhecimentos socialmente úteis com } \\
\text { os públicos desfavorecidos. } \\
\text { 3. Promoção da inter e da transdisciplinaridade. }\end{array}$ & $\begin{array}{l}\text { Eixo não } \\
\text { aplicado } \\
\text { aos } \\
\text { discentes* }\end{array}$ & $\begin{array}{l}\text { Questões } \\
31 \text { a } 50\end{array}$ \\
\hline $\begin{array}{l}\text { Participação } \\
\text { social }\end{array}$ & $\begin{array}{l}\text { 1. Integração da formação acadêmica com a projeção social. (articulação } \\
\text { da extensão com a pesquisa e o ensino). } \\
\text { 2. Luta contra o assistencialismo e paternalismo no serviço universitário } \\
\text { para a comunidade. } \\
\text { 3. Participação ativa na agenda local e nacional de desenvolvimento. }\end{array}$ & $\begin{array}{c}\text { Questões } \\
31 \text { a } 40\end{array}$ & $\begin{array}{l}\text { Questões } \\
51 \text { a } 70\end{array}$ \\
\hline
\end{tabular}

Fonte: Adaptado de Vallaeys, Cruz e Sasia (2009, p. 80-81).

* O modelo prevê que para os estudantes não seja aplicado o eixo Gestão Social do Conhecimento, já que de acordo com Vallaeys, Cruz e Sasia (2009, p. 39 e 50) ele se dirige aos docentes investigadores, ou seja, para quem propõem e coordenada as pesquisas.

Após a validação das medidas dos construtos pelos testes de KMO e esfericidade de Bartlett, procedeu-se a análise de Regressão Múltipla, sendo esta um método de análise apropriado quando se possui uma variável (construto) dependente considerada relacionada a duas ou mais variáveis (construto) independentes métricas. Também realizou-se o teste de análise de Variância ANOVA a fim de ver as relações existentes entre a variável dependente e as variáveis independentes do Modelo de Regressão. Para a validação da regressão e verificação do seu poder de explicação utilizou-se do valor $R 2$ ajustado. Salienta-se que os aspectos de autocorrelação e multicolinearidade foram verificados via Teste de DurbinWatson e Teste de Tolerância e VIF. O estudo, além de verificar se o Modelo de Vallaeys, Cruz e Sasia (2009) é capaz de descrever a percepção de docentes e discentes a respeito da responsabilidade social universitária, busca verificar as seguintes hipóteses de pesquisa:

H0: O Eixo Campus Responsável tem impacto igual na percepção de docentes e discentes.

H1: O Eixo Campus Responsável tem impacto diferente na percepção de docentes e discentes. 
Destaca-se que o Eixo Campus Responsável, tem se destacado na literatura (VÁZQUEZ; AZA; LANERO, 2014; LÓPEZ-NORIEGA; ZALTHEN-HERNÁNDEZ; CERVANTES-ROSAS, 2016; GÓMEZ; ALVARADO; PUJOL, 2018) como um dos eixos mais importantes do modelo de Vallaeys, Cruz e Sasia (2009) e trata-se de um eixo predominante no instrumento de coleta de dados tanto de docentes quanto discentes.

Por fim, ressalta-se que os preceitos éticos da pesquisa consideraram a Resolução $n^{\circ}$ 196 do Conselho Nacional de Saúde, que trata das pesquisas envolvendo seres humanos (BRASIL, 1996), sendo o estudo submetido e aprovado pelo Comitê de Ética em Pesquisa com Seres Humanos da UFFS e da Universidade Estadual do Oeste do Paraná (UNIOESTE) e, no decorrer da coleta de dados, foi solicitado aos participantes que assinalassem seu consentimento por meio do Termo de Consentimento Livre e Esclarecido.

\section{ANÁLISE E DISCUSSÃO DOS RESULTADOS}

A partir da aplicação do questionário com discentes e docentes da UFFS são apresentados os resultados da análise descritiva e da regressão múltipla que visa identificar a percepção de cada grupo sobre a RSU praticada na UFFS.

\subsection{ANÁLISE DESCRITIVA}

$\mathrm{Na}$ análise das maiores médias (escala entre 1. Discordo totalmente a 5. Concordo totalmente) para os discentes destacam-se: a Variável 21 - “A universidade me oferece uma educação ética e cidadã que me ajuda a ser uma pessoa socialmente responsável” $(4,25)$ - do Eixo Formação Profissional e Cidadã; a Variável 35 - Existe uma política explícita na universidade de acesso à educação acadêmica a grupos marginalizados (população indígena, minorias raciais, estudantes de baixa renda, etc.) por meio de bolsas de estudo ou outros meios" (4,23) - presente no Eixo Participação Social e a Variável 22 - "Meu curso é realmente integral, humano e profissional, e não apenas especializado" $(4,20)$ - também do Eixo Formação Profissional e Cidadã. Este dado revela que na percepção dos estudantes da UFFS, a RSU aparece em maior nível de concordância especialmente no Eixo Formação Profissional e Cidadã.

Este eixo, de acordo com Vallaeys, Cruz e Sasia (2009) abarca a gestão socialmente responsável da formação acadêmica (em seu tema, organização curricular, metodologia e proposta didática), que deve estar direcionada para promover competências de responsabilidade em seus graduados, tendo uma relação estreita com os problemas reais 
(econômicos, sociais, ecológicos) da sociedade e o contato com os atores externos envolvidos com esses problemas. O eixo abrange, ainda, as políticas de inclusão, a formação com pensamento crítico e autônomo, a promoção da empregabilidade e aprendizagem ao longo da vida, a promoção do sucesso educativo e combate ao abandono, a integração da aprendizagem baseada em projetos sociais e voluntariado solidário, além da promoção da mobilidade e da colaboração, nacionais e internacionais (ORSIES, 2018).

Já na análise das menores médias para os discentes encontram-se: a Variável 4 - "Eu não percebo discriminação por gênero, raça, status socioeconômico ou orientação política ou sexual" (2,66) presente no Eixo Campus Responsável; a Variável 37 - "No curso de meus estudos, pude constatar que a assistência e o desenvolvimento não estão muito relacionados" $(3,04)$ constante no Eixo Participação Social; e a Variável 12 - "Os gestores tomam as grandes decisões de maneira democrática e consensual" $(3,21)$ oriunda do Eixo Campus Responsável. Logo pode-se inferir que entre os estudantes a UFFS apresenta problemas quanto ao Eixo Campus Responsável, neste eixo observa-se ações que contempla os direitos humanos, a equidade de gênero e não discriminação, bem como a transparência nas decisões da Universidade e sua divulgação a todos os usuários, conforme determina Vallaeys, Cruz e Sasia (2009).

Esse resultado corrobora com o identificado por López-Noriega, Zalthen-Hernández e Cervantes-Rosas (2016) quando estudaram a percepção dos estudantes da UNACAR sobre as ações e práticas de RS. Ao aplicarem as médias das respostas para o Eixo Campus Responsável, observaram que a gestão da RSU era uma área que necessitava de mais atenção por parte da instituição, ou seja, deveria ser vista como área de oportunidade para aperfeiçoar e atingir um nível mais satisfatório entre os alunos.

$\mathrm{Na}$ análise das maiores médias para os docentes, destacam-se: a Variável 47 - "Os estudantes de graduação devem necessariamente praticar pesquisas durante a sua formação (4,35) - Eixo Gestão Social do Conhecimento; a Variável 26 - "Eu costumo ligar os conteúdos temáticos ensinados aos problemas sociais e ambientais de hoje" $(4,30)$ do Eixo Formação Profissional e Cidadã; e a Variável 13 - "Os gestores da universidade foram escolhidos democraticamente e transparentemente" $(4,16)$ presente no Eixo Campus Responsável. Observa-se que os docentes destacaram mais Eixos de RSU do que os estudantes.

Com as menores médias para os docentes, são evidenciadas: a Variável 29 - "Na minha especialidade, temos reuniões com atores sociais externos para discutir a relevância 
social do currículo" (2,56) do Eixo Formação Profissional e Cidadã; a Variável 11 - "O pessoal docente e não docente recebe formação em questões ambientais da universidade" $(2,84)$ do Eixo Campus Responsável; e a Variável 22 - "Reuniões são realizadas com colegas para examinar os aspectos de responsabilidade social ligados à carreira que ensino" $(2,95)$ também do Eixo Formação Profissional e Cidadã.

Observa-se desta forma que os menores desempenhos da Instituição para os docentes residem especialmente no Eixo Formação Profissional e Cidadã, este fato corrobora com a análise documental dos Projetos Pedagógicos Curriculares do Curso de Graduação (PPCs), onde se aponta a perspectiva de que os projetos estão perdendo a ideia de uma formação humana e social, uma vez que componentes curriculares, como o de Direito e Cidadania, foi eliminado da grade curricular de alguns cursos. No relatório da II COEPE este dado também foi apontado pelos docentes.

Após análise descritiva das variáveis que compõem os instrumentos de coleta de dados procedeu-se as médias de cada constructo, sendo estabelecido com base na literatura os seguintes construtos:

1) Para discentes são construtos independentes: Participação Social, Formação Profissional e Cidadã e Campus Responsável; e Construto dependente: UFFS é uma instituição com compromisso social.

2) Para os docentes são construtos independentes: Participação Social, Formação Profissional e Cidadã, Campus Responsável e Gestão Social do Conhecimento; Construto dependente: UFFS é uma instituição com compromisso social.

\subsection{ANÁLISE DE REGRESSÃO}

Antes de calcular as estatísticas do teste de regressão múltipla, procurou-se, primeiramente determinar se as medidas dos constructos são significantemente correlacionadas. Os testes mais amplamente usados para esse fim são os testes de KaiserMeyer-Olkin (KMO) e esfericidade de Bartlett. O teste de KMO representa a razão das correlações ao quadrado entre as variáveis, com parâmetros recomendados entre 0 e 1, sendo que quanto mais próximo de 1 melhor o resultado (FIELD, 2009), e o teste de significância geral de Esfericidade de Bartlett que examina as correlações entre todos os construtos e avalia se existe, coletivamente, intercorrelações significativas, sendo que, recomenda-se $p<0,05$ (HAIR. Jr. et al., 2005).

Os dados indicam intercorrelações entre os construtos dentro dos parâmetros da literatura, sendo estas significativas. Destaca-se para os dados dos discentes: KMO: ,814 e 
Esfericidade de Bartlett com sig=,000; e os dados dos docentes KMO: ,844 e Esfericidade de Bartlett com sig $=, 000$.

Após a validação das medidas dos construtos pelos testes de KMO e esfericidade de Bartlett, procedeu-se a análise de Regressão Múltipla, sendo esta um método de análise apropriado quando se possui uma variável (construto) dependente considerada relacionada a duas ou mais variáveis (construtos) independentes métricas. O objetivo da análise de regressão múltipla é prever as mudanças na variável (construto) dependente como resposta a mudanças nas variáveis (construtos) independentes. Esse objetivo é alcançado, por meio da regra estatística dos mínimos quadrados (HAIR JR. et al., 2005; DANCEY; REIDY, 2006).

Para tanto, procedeu-se a análise de Variância ANOVA. Um teste ANOVA sugere que seja significativo $(\operatorname{sig}<0,05)$, e que as estimativas independentes da variância para a variável dependente reflitam a variabilidade dentro dos grupos e que represente as diferenças entre grupos atribuíveis aos efeitos de tratamento dos dados (HAIR JR. et al., 2005), sendo que a estatística $F$ é a razão entre variância entre os grupos pela variância dentro do grupo (DANCEY; REIDY, 2006). As Tabelas 2 e 3, apresentam as estatísticas da Análise de Variância (ANOVA) para os dois grupos analisados - discentes e docentes.

Tabela 2 Análise de Variância (ANOVA) ${ }^{\mathrm{b}}$ - discentes

\begin{tabular}{|c|c|c|c|c|c|}
\hline Modelo & Soma dos quadrados & Graus de liberdade & Média quadrática & $\mathbf{F}$ & Sig. \\
\hline Regressão & 18,182 & 3 & 6,061 & 32,858 &, $000^{\mathrm{a}}$ \\
\hline 1 Residual & 23,241 & 126 &, 184 & & \\
\hline Total & 41,423 & 129 & & & \\
\hline $\begin{array}{l}\text { a. Construtos } \\
\text { Responsável. } \\
\text { b. Construto d }\end{array}$ & $\begin{array}{l}\text { oendentes: (Constante) } \\
\text { ente: UFFS é uma instit }\end{array}$ & $\begin{array}{l}\text { articipação Social, } \\
\text { ão com compromisso }\end{array}$ & nação Profissional & Cidadã, & ampus \\
\hline
\end{tabular}

Fonte: Elaborada pelas autoras.

Analisando-se os resultados do teste de Variância (ANOVA) para os discentes percebe-se que ele apresenta probabilidade de que as diferenças sejam significativas $F=32,85$ e sig=,000, o que valida o modelo.

Tabela 3 Análise de Variância (ANOVA) ${ }^{\mathrm{b}}$ - docentes

\begin{tabular}{|c|c|c|c|c|c|}
\hline Modelo & Soma dos quadrados & Graus de liberdade & Média quadrática & $\mathbf{F}$ & Sig. \\
\hline \multirow[t]{3}{*}{ Regressão } & 13,739 & 4 & 3,435 & 22,476 &, $000^{\mathrm{a}}$ \\
\hline & 8,864 & 58 & ,153 & & \\
\hline & 22,603 & 62 & & & \\
\hline $\begin{array}{l}\text { a. Construtos } \\
\text { Responsável, } \\
\text { b. Construto d }\end{array}$ & $\begin{array}{l}\text { dependentes: (Constant } \\
\text { ão Social do Conhecim } \\
\text { adente: UFFS é uma ins }\end{array}$ & $\begin{array}{l}\text { Participação Social, } \\
\text { o. } \\
\text { ição com compromiss }\end{array}$ & $\begin{array}{l}\text { mação Profissional } \\
\text { iial. }\end{array}$ & Cidadã, & ampus \\
\hline
\end{tabular}

Fonte: Elaborada pelas autoras. 
Da mesma forma, analisando-se os resultados do teste de Variância (ANOVA) para os docentes percebe-se que também apresenta probabilidade de que as diferenças sejam significativas $\mathrm{F}=22,476$ e sig=,000. Nas Tabelas 4 e 5, estão dispostos os resultados de validação e poder explicativo da análise de Regressão Múltipla, que envolve o exame do valor $R 2$ ajustado.

Para validar o modelo de regressão, apresenta-se o teste de ausência de autocorrelação serial, por meio do teste de Durbin-Watson. A ausência de autocorrelação serial pressupõe que os resíduos são independentes entre si e só se observa o efeito das variáveis (construtos) independentes sobre a variável (construto) dependente, sendo que, busca-se a inexistência de autocorrelação residual (CORRAR; PAULO; DIAS FILHO, 2012; GUJARATI; PORTER, 2011). Segundo Gujarati e Porter (2011), os limites de $d$ devem estar entre 0 e 4.

Tabela 4 Estimativa do modelo a e teste de Durbin-Watson - discentes

\begin{tabular}{llcccc}
\hline Modelo & $\mathbf{R}$ & $\mathbf{R}^{2}$ & $\mathbf{R}^{2}$ ajustado & Erro-padrão da estimativa & Durbin-Watson \\
\hline 1 &, 663 & 439 &, 426 &, 42948 & 2,010 \\
\hline $\begin{array}{l}\text { a. Construtos } \\
\text { Responsável. Independentes: }\end{array}$ & (Constante), Participação & Social, Formação Profissional e Cidadã, Campus \\
b. Construto dependente: UFFS é uma instituição com compromisso social. & \\
\hline
\end{tabular}

Fonte: Elaborada pelas autoras.

Os resultados (Tabela 4) do teste de Durbin-Watson $(2,010)$ para os dados coletados junto aos discentes indica que os valores atendem os pressupostos previstos na literatura (GUJARATI; PORTER, 2011). Também indicou o resultado da estimativa do ajuste geral do modelo de regressão para os dados coletados com os discentes e apresentou um $\mathrm{R}^{2}$ ajustado de ,426 indicando que a variabilidade do construto dependente - UFFS é uma instituição com compromisso social - foi explicada em $42,60 \%$ pelos construtos independentes consideradas significativas $(\mathrm{p}<0,05)$. Na Tabela 5 são apresentados os resultados da estimativa dos docentes.

Tabela 5 Estimativa do modelo ${ }^{\mathrm{a}}$ e teste de Durbin-Watson - docentes

\begin{tabular}{llcccc}
\hline Modelo & $\mathbf{R}$ & $\mathbf{R}^{\mathbf{2}}$ & $\mathbf{R}^{\mathbf{2}}$ ajustado & Erro-padrão da estimativa & Durbin-Watson \\
\hline 1 &, 780 &, 608 &, 581 &, 39093 & 1,918 \\
\hline a. Construtos & Independentes: (Constante), Participação & Social, Formação Profissional e Cidadã, Campus \\
Responsável, Gestão Social do Conhecimento. & & \\
b. Construto dependente: UFFS é uma instituição com compromisso social. &
\end{tabular}

Fonte: Elaborada pelas autoras.

Os resultados do teste de Durbin-Watson $(1,918)$ para os dados coletados junto aos docentes também indicam que os valores atendem os pressupostos previsto na literatura 
(GUJARATI; PORTER, 2011), sendo que, o resultado da estimativa do ajuste geral do modelo de regressão para os dados coletados com os docentes apresentou um $\mathrm{R}^{2}$ ajustado de ,581 indicando que a variabilidade do construto dependente - UFFS é uma instituição com compromisso social - foi explicada em $58,10 \%$ pelos construtos considerados significativos.

As Tabelas 6 e 7 apresentam os construtos independentes que explicam o construto dependente. Nessas tabelas estão expostos os testes de Tolerância e Fator de Inflação da Variância (VIF) para análise de multicolinearidade. Destaca-se ser essencial a verificação de multicolineariedade pois construtos altamente colineares podem distorcer os resultados substancialmente ou torná-los muito instáveis (HAIR JR. et al., 2005).

O Teste de Tolerância e VIF indicam em que grau cada construto dependente é explicado pelos demais construtos independentes. Valores de VIF até 1 não indicam multicolinearidade, de 1 a 10 aceitável, e acima de 10 problemática, assim, procura-se valores VIF pequenos como indicativos de baixa intercorrelação entre as variáveis. Para corroborar, o Teste de Tolerância superior a 1 não indica multicolinearidade, de 1 a 0,10 indicam multicolinearidade aceitável e abaixo de 0,10 indicam multicolinearidade problemática (CORRAR; PAULO; DIAS FILHO, 2012; HAIR JR. et al., 2005). A Tabela 6 demonstra os coeficientes do Modelo e os Testes de Tolerância e VIF na amostra de discentes.

Tabela 6 Coeficientes do Modelo de Regressão e testes de Tolerância e VIF - discentes

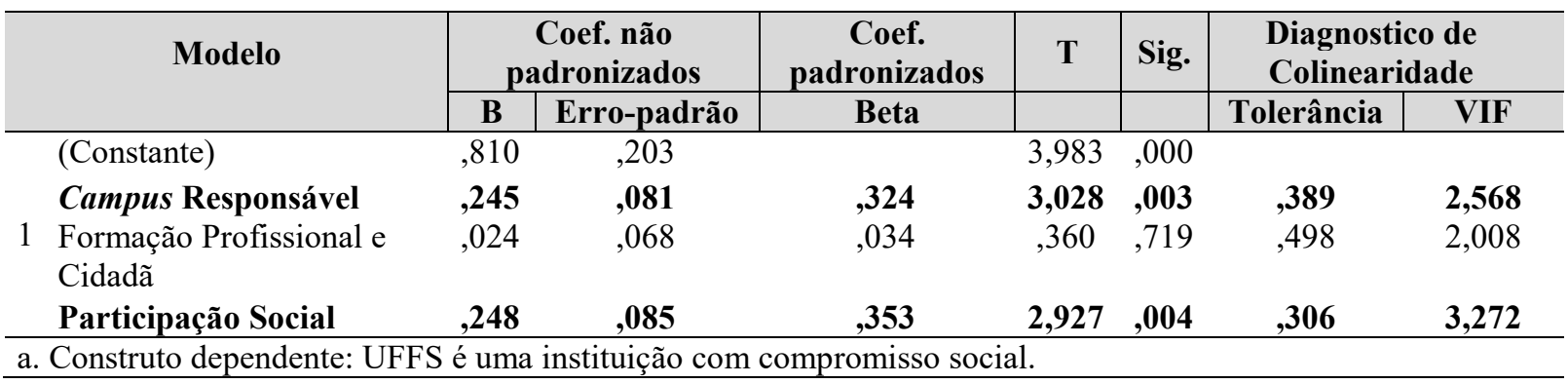

Fonte: Elaborada pelas autoras.

Os dados da Tabela 6 indicam que não encontrou-se evidência da existência de multicolinearidade nesses resultados de regressão, como indicado pelas medidas de Tolerância e VIF (Tolerância estão entre 1 e 0,10 e VIF estão entre 1 e 10) e que os resultados indicam que a interpretação dos coeficientes da variável estatística de regressão não foi afetada desfavoravelmente pela multicolinearidade (HAIR Jr. et al., 2005).

$\mathrm{Na}$ análise dos coeficientes do Modelo de Regressão para os dados coletados com os discentes destaca-se que os constructos com maior poder explicativo sobre o constructo 
dependente (UFFS é uma instituição com compromisso social) foram: Participação Social (Beta=,353 e sig=0,004) e Campus Responsável (Beta=,324 e sig=,003), respectivamente. Formação Profissional e Cidadã não tem poder explicativo significativo sobre o construto dependente. A Tabela 7 apresenta o resultado do modelo de regressão na visão dos docentes.

Tabela 7 Coeficientes do Modelo de Regressão e testes de Tolerância e VIF - docentes

\begin{tabular}{|c|c|c|c|c|c|c|c|c|}
\hline \multirow{2}{*}{\multicolumn{2}{|c|}{ Modelo }} & \multicolumn{2}{|c|}{$\begin{array}{c}\text { Coef. não } \\
\text { padronizados }\end{array}$} & \multirow{2}{*}{$\begin{array}{c}\begin{array}{c}\text { Coef. } \\
\text { padronizados }\end{array} \\
\text { Beta }\end{array}$} & \multirow{2}{*}{$\mathbf{T}$} & \multirow{2}{*}{ Sig. } & \multicolumn{2}{|c|}{$\begin{array}{l}\text { Diagnostico de } \\
\text { Colinearidade }\end{array}$} \\
\hline & & B & $\begin{array}{l}\text { Erro- } \\
\text { padrão }\end{array}$ & & & & Tolerância & VIF \\
\hline \multirow{5}{*}{1} & (Constante) & ,441 & 262 & & 1,684 & ,098 & & \\
\hline & Campus Responsável & ,344 & ,104 & ,486 & 3,318 &, 002 & ,315 & 3,177 \\
\hline & $\begin{array}{l}\text { Formação Profissional } \\
\text { e Cidadã }\end{array}$ &,- 100 & ,092 &,- 145 & $-1,085$ & ,282 & ,378 & 2,649 \\
\hline & $\begin{array}{l}\text { Gestão Social do } \\
\text { Conhecimento }\end{array}$ & ,330 & ,127 & ,406 & 2,606 & ,012 & ,278 & 3,593 \\
\hline & Participação Social &, 050 &, 100 &, 062 &, 501 & ,618 & ,445 & 2,246 \\
\hline
\end{tabular}

Fonte: Elaborada pelas autoras.

Os dados indicam que não foram encontradas evidências da existência de multicolinearidade nesses resultados de regressão, como indicado pelas medidas de Tolerância e VIF (Tolerância estão entre 1 e 0,10 e VIF estão entre 1 e 10). Além disso, os resultados apontam que a interpretação dos coeficientes da variável estatística de regressão não foi afetada desfavoravelmente pela multicolinearidade (HAIR Jr. et al., 2005).

$\mathrm{Na}$ análise dos dados coletados com os docentes o coeficiente do Modelo de Regressão destaca-se que os constructos com maior poder explicativo sobre o constructo dependente (UFFS é uma instituição com compromisso social) foram: Campus responsável (Beta=,486 e sig=0,02) e Gestão Social do Conhecimento (Beta=,406 e sig=,012). Formação Profissional e Cidadã e Participação Social não tiveram poder explicativo significativo sobre o construto dependente - UFFS é uma instituição com compromisso social.

Observa-se a partir das duas regressões (discentes e docentes) que o construto independente Campus Responsável explica significativamente a percepção que docentes e discentes possuem a respeito da responsabilidade social da UFFS e deve ser destacado no âmbito do Modelo Proposto por Vallaeys, Cruz e Sasia (2009). Salienta-se que o objetivo do Eixo Campus Responsável é reafirmar os valores diários e promover um comportamento organizacional responsável por parte de todos os membros da comunidade universitária.

O estudo de Gómez, Alvarado e Pujol (2018) pode ser destacado como determinante, pois ao verificarem a perspectiva de 356 estudantes, 99 funcionários administrativos e 78 
docentes sobre a RSU de uma universidade privada em Porto Rico, por meio da aplicação de média e desvio padrão sobre as respostas dos questionários, visualizaram que os três grupos apontaram o Campus Responsável como eixo mais significativo de desenvolvimento da RSU.

Apesar do que foi alcançado na área da RSU nos últimos anos, a julgar pelos resultados dessa pesquisa, deve-se continuar insistindo no reconhecimento e na sistematização da responsabilidade social nas universidades, para que se torne reconhecida em todos os âmbitos institucionais: na gestão, no ensino, na pesquisa e na extensão, pois como destacam Vallaeys, Cruz e Sasia (2009), um dos valores importantes que toda universidade deve cuidar é, justamente, a coerência institucional. Essa é a completa coincidência entre ação e discurso institucional e consistência entre as áreas da universidade. Ao assumirem a RS, as universidades precisam ser capazes de um processo permanente de melhoria que não poderá ser alcançado em sua totalidade.

\section{CONSIDERAÇÕES FINAIS}

A Responsabilidade Social Universitária é uma dimensão que relaciona vários aspectos quanto aos conhecimentos produzidos e difundidos pela universidade pública em seus diferentes contextos sociais e que repercute na vida da comunidade acadêmica e do entorno em qual ela está inserida. Desta forma, descrever a concepção dos docentes e discentes da Universidade Federal da Fronteira Sul (UFFS) a respeito desta foi o objetivo deste estudo, que utilizou como modelo de análise a proposta de RSU desenvolvida por Vallaeys, Cruz e Sasia (2009).

Neste sentido, um questionário do tipo escala Likert foi aplicado com 133 discentes e 65 docentes da UFFS e diversos testes estatísticos foram realizados a fim de identificar a concepção de cada um dos grupos. Os resultados da análise descritiva revelam que o Eixo Campus Responsável tem maior impacto na percepção de responsabilidade social da UFFS pelos docentes do que pelos discentes, o que nos leva a refutar a hipótese $\mathrm{H} 0$ e aceitar a hipótese H1.

Para os discentes, a regressão múltipla revelou que os constructos com maior poder explicativo sobre o constructo dependente (UFFS é uma instituição com compromisso social) foram: Participação Social e Campus Responsável, respectivamente. Desta forma, pode-se afirmar que na visão dos estudantes os aspectos relacionados a integração da formação acadêmica com o projeto social da Universidade, bem como participação ativa na agenda 
local e nacional da Instituição e o projeto social desenvolvimento pela IES são vertentes importantes do compromisso social da IES.

Já na visão dos docentes o coeficiente do modelo de regressão destacou que os constructos com maior poder explicativo sobre o constructo dependente foram: Campus Responsável e Gestão Social do Conhecimento. Para os professores, além dos aspectos que permeiam o Eixo Campus Responsável, são determinantes também para o compromisso social a disseminação de conhecimento, pesquisa e modelos epistemológicos promovidos pela Universidade.

Ressalta-se que apesar dos grupos (docentes e discentes) apresentarem visões diferentes sobre o compromisso social da UFFS, o Eixo Campus Responsável é um eixo comum na visão de ambos os grupos e merece destaque no Modelo de RSU de Vallaeys, Cruz e Sasia (2009). Neste sentido, é importante que, as IES atentem para integrar as preocupações de responsabilidade social de forma transversal na estratégia e na matriz base das atividades desenvolvidas.

Por fim, esta análise contribui para o entendimento da responsabilidade social universitária, pois é considerado um tema abrangente e complexo. Indo além, auxilia na ampliação do uso do Modelo de RSU no Brasil, especialmente por meio de métodos quantitativos de análise. Como limitação do método, destaca-se a dificuldade de inferir generalizações, uma vez que os resultados obtidos podem não se estender a outros casos. Além disso, o estudo não abrigou a concepção de toda a comunidade acadêmica, o que pode ser inclusive objeto de pesquisas futuras na IES e em outras.

\section{REFERENCIAS}

BOHRER, Iza N. et al. A história das universidades: o despertar do conhecimento. In: Anais da $14^{a}$ Jornada Nacional de Educação: a educação na sociedade dos meios virtuais, Santa Maria, v. 1, n. 1, p. 1-10, 2008.

BRASIL. Presidência da República. Casa Civil. Lei no 10.861, de 14 de abril de 2004. Institui o Sistema Nacional de Avaliação da Educação Superior - SINAES e dá outras providências. Brasília, Diário Oficial [da] República Federativa do Brasil, Brasília, DF, 15 abr. 2004. Seção, p. 3. Disponível em: http://www.planalto.gov.br/ccivil_03/_ato20042006/2004/lei/110.861.htm. Acesso em: 02 maio 2020.

BRASIL. Presidência da República. Casa Civil. Lei n 9.394, de 20 de dezembro de 1996. Lei de Diretrizes e Bases da Educação Nacional. Diário Oficial da União, Brasília, DF, 24 dez. 1996. Disponível em: http://www.planalto.gov.br/ccivil_03/Leis/L9394.htm. Acesso em: 02 maio 2020. 
CALDERÓN, Adolfo Ignacio. Responsabilidade social: desafios à gestão universitária. Estudos: Revista da Associação Brasileira de Mantenedores do Ensino Superior (ABMES), Brasília, n. 34, ano 23, p. 13-27, 2005.

CARDENUTO, Raquel Matys et al. As Atividades de Extensão como Compromisso Social: Um Estudo de Caso no Instituto Federal de Santa Catarina (IFSC) e na Fundação Universidade do Estado de Santa Catarina (UDESC). Revista GUAL, Florianópolis, v. 10, n. 4, pp. 134-156, Edição Especial 2017.

CÉSPEDES AGUIRRE, Lourdes Lucila. Percepción de los grupos de interés de la Responsabilidad Social Universitaria en una universidad pública. Revista Conrado, v. 15, n. 70, pp. 81-89, 2019.

CORRAR, Luiz J.; PAULO, Edilson; DIAS FILHO, José Maria (Coord.). Análise multivariada: para os cursos de administração, ciências contábeis e economia. 1. ed. São Paulo: Atlas, 2012.

CRUZ, Rosilene Maria; PASSOS, Elizabete S. Inserção da Responsabilidade Social nas IES: caminhos para o desenvolvimento humano. In: ROCHA, Niveia Maria Fraga; RABELO, Patrícia Fraga Rocha (Orgs.). Educação, Desenvolvimento Humano e Responsabilidade Social: Fazendo Recortes na Multidisciplinaridade. Salvador: Fast Design, 2010, p. 117-137.

DANCEY, Christine P., REIDY, John. Estatística sem matemática para psicologia usando SPSS para Windows. Porto Alegre: Artes Médicas, 2006.

DANESI, Luiz Carlos; SIQUEIRA, Marino da Silva. Identidade Institucional e sua relação com a profissionalização da gestão. In: KÖCHE, José Carlos; BUOGO, Ana Lúcia (Orgs.). Desafios da Gestão Universitária. Caxias do Sul: EDUCS, 2014, p. 29-52.

DUEÑAS, Maira Alejandra Sissa. Responsabilidad social universitaria: una mirada desde América Latina y Colombia. Inquietud Empresarial, v. XV, n. 2, p. 65-85, 2015.

DUQUE, Pedro; CERVANTES-CERVANTES, Luis-Salvador. Responsabilidad Social Universitaria: una revisión sistemática y análisis bibliométrico. Estudios Gerenciales, v. 35, n. 153, p. 451-464, 2019.

GIL, Antônio Carlos. Como elaborar projetos de pesquisa. 5. ed. São Paulo: Atlas, 2010.

GÓMEZ, Lina M.; ALVARADO NAVEIRA, Yanitzary; PUJOL BERNABEL, Aileen. Practicando la Responsabilidad Social Universitaria en el Caribe: Perspectivas de los públicos internos. Revista Digital de Investigación en Docencia Universitaria, v. 12, n. 1, pp. 96$118,2018$.

GOMEZ, Lina M.; PRECIADO, Lucely Vargas. CSR trends in the top 100 US business schools: A theory practice relationship. In: Crowther, D. (Eds.) Education and corporate social responsibility international perspectives. Developments in Corporate Governance and Responsibility. Bingley, UK: Emerald Group Publishing Limited, 2013. 
GUJARATI, Damodar N.; PORTER, Dawn C. Econometria básica. 5. ed. Porto Alegre: AMGH, 2011.

HAIR JR., Joseph F.; ANDERSON, Rolph E.; TATHAM, Ronald L.; BLACK, William C. Análise multivariada de dados. 5. ed. Porto Alegre: Bookman, 2005.

JIMÉNEZ, Clara Guijarro; MARTÍNEZ, Antonio Gomera; LÓPEZ, Miguel Antúnez. "Propuesta de indicadores de la Responsabilidad Social Universitaria conforme a la Guía G4 del GRI: el caso de la Universidad de Córdoba", CIRIEC-España, Revista de Economía Pública, Social y Cooperativa, v. 87, p. 103-137, 2016.

KISZNER, Sabrina de Farias Borba. Responsabilidade social universitária sob a perspectiva da avaliação institucional: uma análise à luz da gestão integral e transversal em instituições comunitárias de educação superior. 2018, 147f. Dissertação (Mestrado do Programa de Pós-Graduação em Desenvolvimento Regional das Faculdades Integradas de Taquara-RS) Taquara-RS, 2018.

LARRÁN-JORGE, Manuel, et al. Análisis del nivel de implantación de políticas de responsabilidad social en las universidades españolas. Fundación Carolina: Madrid, 2012.

LO, Carlos Wing-Hung et al. University Social Responsibility: Conceptualization and an Assessment Framework.In: SHEK, Daniel T. L.; HOLLISTER, Roberto M. University Social Responsibility and Quality of Life: A Global Survey of Concepts and Experiences. v. 8. [S.1]: Springer Nature Singapore, 2017. p. 37-59.

LÓPEZ-NORIEGA, Myrna Delfina; ZALTHEN - HERNÁNDEZ, Lorena; CERVANTES ROSAS, María de los Ángeles. La responsabilidad social universitaria desde la perspectiva del alumno. Ra Ximhai, v. 12, n. 6, pp. 305-314, julio-diciembre, 2016.

MARCONI, Marina de Andrade; LAKATOS, Eva Maria. Técnicas de pesquisa: planejamento e execução de pesquisas, amostragens e técnicas de pesquisas, elaboração, análise e interpretação de dados. 5 ed. São Paulo: Atlas, 2002, 282 p.

MEBARACK, William Antonio Geliz; RUIZ, Rodolfo Antonio Tejada. Buena práctica de RSU em gestión social del conocimiento y participación social: Proyecto "Inventa Diseño" Utec, elaboración de manuales de identidad corporativa. In: VILLA, Estela De (Coord.). Responsabilidad social de las universidades: Tomo VII. 1. ed. Buenos Aires: Fundación Red Latinoamericana de Cooperación Universitaria - RLCU, 2018.

NASCIMENTO, José Mancinelli Ledô do et al. Metodologia para avaliar a responsabilidade social das universidades públicas. Avaliação, Campinas, Sorocaba-SP, v. 20, n. 3, p. 685-702, nov. 2015.

OBSERVATÓRIO DA RESPONSABILIDADE SOCIAL E INSTITUIÇÕES DE ENSINO SUPERIOR (ORSIES). Livro verde sobre Responsabilidade social e instituições de ensino superior. ORSIES: março 2018. Disponível em: http://orsies.forum.pt/. Acesso em: 5 set. 2018. 
OLIVEN, Arabela Campos. Histórico da Educação Superior no Brasil. In: SOARES, M. S. A. (Org.). A Educação Superior no Brasil. Brasília: Coordenação de Aperfeiçoamento de Pessoal de Nível Superior (CAPES), 2002.

PUCCI, Bruno. A indissociabilidade entre Ensino Pesquisa e Extensão. Impulso, Piracicaba, p. 33-42, 1991.

QUEZADA, Gaete R. La responsabilidad social universitaria como desafío para la gestión estratégica de la educación superior: el caso de espana. Revista de Educación, v. 355, pp. 109-133, Mayo-agosto 2011.

REIS, Antônio Ribas. Gestão da responsabilidade social: estudo em universidade da região metropolitana de Salvador. 2007. 171f. Dissertação (Mestrado em Gestão do

Desenvolvimento Local Sustentável) - Fundação Visconde de Cairu, Salvador, 2007.

RICHARDSON, Roberto Jarry. Pesquisa Social: métodos e técnicas. São Paulo: Atlas, 1999.

ROSETTO, Marcia Regina Chrispim Alvares. Instituições de ensino superior e responsabilidade social: um estudo sobre as representações de lideranças da educação superior brasileira. 2011. 131f. Tese (Doutorado em Educação) - Pontifícia Universidade Católica de São Paulo, São Paulo, 2011.

SANTELI, Genoveva Espinoza; MONTOYA, Marco Guachamín. La responsabilidad social universitaria en Ecuador. Estudios de la Gestión: revista internacional de administración, n. 1, p. 9-27, enero-junio 2017.

SIDOROVA, Luis. Responsabilidad Social y Gestión Organizacional en La Universidad de Carabobo: Sincronismo Ineludible en Cambios Coyunturales. Revista Multidisciplinaria Dialógica, v. 12, n. 1, p. 208-234, Enero-Junio 2015.

UNIVERSIDADE FEDERAL DA FRONTEIRA SUL (UFFS). A Instituição Apresentação. Disponível em:

https://www.uffs.edu.br/institucional/a_uffs/a_instituicao/apresentacao. Acesso em: 27 abril 2020 .

UNIVERSIDADE FEDERAL DA FRONTEIRA SUL (UFFS). Dados Abertos. Estudantes ingressantes da graduação. Disponível em: https://dados.uffs.edu.br/dataset. Acesso em: 16 jul. 2018a.

UNIVERSIDADE FEDERAL DA FRONTEIRA SUL (UFFS). Graduação - Cursos.

Disponível em: https://www.uffs.edu.br/institucional/pro-reitorias/graduacao/cursos. Acesso em: 28 ago. 2018 b.

UNIVERSIDADE FEDERAL DA FRONTEIRA SUL (UFFS). Pós-Graduação - Mestrado. Disponível em: https://www.uffs.edu.br/institucional/pro-reitorias/pesquisa-e-posgraduacao/pos-graduacao/especializacao. Acesso em: 28 ago. 2018c.

VALLAEYS, François. Les fondements éthiques de la Responsabilité Sociale [en línea] /Tesis doctoral sostenida. 5 diciembre 2011. Disponível em: 
http://blog.pucp.edu.pe/media/410/20111214les_fondements_ethiques_de_la_ responsabilite_sociale_francois_valleys.pdf. Acesso em: 15 março 2020 .

VALLAEYS, François. Introducción a la Responsabilidad Social Universitaria RSU. Barranquilla: Ediciones Universidad Simón Bolívar, 2016.

VALLAEYS, François. Responsabilidade social universitária: uma definição prudente e responsável. Colóquio - Revista do Desenvolvimento Regional, Faccat, Taquara/RS, v. 14, n. 2, jul./dez. 2017.

VALLAEYS, François; CRUZ, Cristina de la; SASIA, Pedro M. Responsabilidad social universitaria: manual de primeros pasos. México: The McGraw-Hill Companies; InterAmerican Development Bank, 2009. Disponível em: http://blog.pucp.edu.pe/blog/wpcontent/uploads/sites/54/2012/05/manual_digital_bid_rsu.pdf. Acesso em: 28 maio 2018.

VÁZQUEZ, José Luis; AZA, Carlota L.; LANERO, Ana. Students' experiences of university social responsibility and perceptions of satisfaction and quality of service. EKONOMSKI VJESNIK, v. XXVIII, p. 25-39, Posebno Izdanje/2014.

VILLALONGA, Yoarnelys Vasallo; PASPUEL, Edison Favio Arciniegas. Desarrollo Sustentable y Responsabilidad Social Em La Educación Superior. Revista San Gregorio, n. 10, v. 2, p. 93-105, Julio-Diciembre, 2015. 